\title{
Evolution of competence and DNA uptake specificity in the
} Pasteurellaceae

\author{
Rosemary J Redfield*1, Wendy A Findlay², Janine Bossé3, J Simon Kroll3, \\ Andrew DS Cameron ${ }^{4}$ and John HE Nash ${ }^{2}$
}

\author{
Address: ${ }^{1}$ Dept. of Zoology, University of British Columbia, Vancouver BC Canada, ${ }^{2}$ Institute for Biological Sciences, National Research Council \\ of Canada, Ottawa ON Canada, ${ }^{3}$ Dept. of Paediatrics, Faculty of Medicine, Imperial College London, London W2 1PG UK and ${ }^{4}$ Dept. of \\ Microbiology and Immunology, University of British Columbia, Vancouver BC Canada \\ Email: Rosemary J Redfield* - redfield@zoology.ubc.ca; Wendy A Findlay - Wendy.Findlay@nrc-cnrc.gc.ca; \\ Janine Bossé - j.bosse@imperial.ac.uk; J Simon Kroll - s.kroll@imperial.ac.uk; Andrew DS Cameron - dafydd@interchange.ubc.ca; \\ John HE Nash - John.Nash@nrc-cnrc.gc.ca \\ * Corresponding author
}

Published: 12 October 2006

BMC Evolutionary Biology 2006, 6:82 doi:10.1186/147|-2/48-6-82
Received: 12 June 2006

Accepted: 12 October 2006

This article is available from: http://www.biomedcentral.com/|47|-2/48/6/82

(c) 2006 Redfield et al; licensee BioMed Central Ltd.

This is an Open Access article distributed under the terms of the Creative Commons Attribution License (http://creativecommons.org/licenses/by/2.0), which permits unrestricted use, distribution, and reproduction in any medium, provided the original work is properly cited.

\begin{abstract}
Background: Many bacteria can take up DNA, but the evolutionary history and function of natural competence and transformation remain obscure. The sporadic distribution of competence suggests it is frequently lost and/or gained, but this has not been examined in an explicitly phylogenetic context. Additional insight may come from the sequence specificity of uptake by species such as Haemophilus influenzae, where a 9 bp uptake signal sequence (USS) repeat is both highly overrepresented in the genome and needed for efficient DNA uptake. We used the distribution of competence genes and DNA uptake specificity in $H$. influenzae's family, the Pasteurellaceae, to examine the ancestry of competence.
\end{abstract}

Results: A phylogeny of the Pasteurellaceae based on 12 protein coding genes from species with sequenced genomes shows two strongly supported subclades: the Hin subclade $(H$. influenzae, Actinobacillus actinomycetemcomitans, Pasteurella multocida, Mannheimia succiniciproducens, and $H$. somnus), and the Apl subclade (A. pleuropneumoniae, M. haemolytica, and $H$. ducreyi). All species contained homologues of all known $H$. influenzae competence genes, consistent with an ancestral origin of competence. Competence gene defects were identified in three species (H. somnus, $H$. ducreyi and $M$. haemolytica); each appeared to be of recent origin.

The assumption that USS arise by mutation rather than copying was first confirmed using alignments of $H$. influenzae proteins with distant homologues. Abundant USS-like repeats were found in all eight Pasteurellacean genomes; the repeat consensuses of species in the Hin subclade were identical to that of $H$. influenzae (AAGTGCGGT), whereas members of the Apl subclade shared the consensus ACAAGCGGT. All species' USSs had the strong consensus and flanking AT-rich repeats of $H$. influenzae USSs. DNA uptake and competition experiments demonstrated that the Apl-type repeat is a true USS distinct from the Hin-type USS: A. pleuropneumoniae preferentially takes up DNA fragments containing the Apl-type USS over both $H$. influenzae and unrelated DNAs, and $H$. influenzae prefers its own USS over the Apl type.

Conclusion: Competence and DNA uptake specificity are ancestral properties of the Pasteurellaceae, with divergent USSs and uptake specificity distinguishing only the two major subclades. The conservation of most competence genes over the $\sim 350$ million year history of the family suggests that lineages that lose competence may be evolutionary dead ends. 


\section{Background}

Many bacteria are able to take up DNA from the environment [1]. DNA provides these naturally competent cells with nutrients (nucleotides, $\mathrm{N}$ and $\mathrm{P}$ ), while recombination of incoming DNA with the cell's genome can also provide new genetic information. However, many aspects of the evolution of competence remain unclear.

Competence is widely distributed among bacteria, and some of the genes required for DNA uptake are shared between even distant relatives, suggesting an ancient common origin for competence. For example, the Gram positive bacteria Bacillus subtilis and Streptococcus pneumoniae and the Gram negative Neisseria gonorrhoeae and Haemophilus influenzae all require homologues of type four pilus proteins and of the ComEC/Rec2 membrane channel [1]. However, the regulatory processes controlling expression of these competence genes are very different in the different organisms [2]. Furthermore the distribution of natural competence is surprisingly sporadic; most naturally competent bacteria have many relatives, including other strains of the same species, that cannot be transformed under laboratory conditions (for examples see [3-6]). Two explanations seem equally plausible. First, competence might be ancestral to most major lineages but frequently lost (and possibly regained, under different regulation). Alternatively, competence might be frequently gained in independent lineages, e.g. if the genetic requirements for DNA uptake are simple and readily met by laterally transferred genes or by mutation of genes with related functions such as those associated with type IV pili.

The uptake specificity of some naturally competent bacteria can also guide inferences about the evolution of competence. Although many naturally competent bacteria will take up DNA fragments from any source with equal efficiency, members of some Gram-negative families take up DNA fragments from their own species much more efficiently than unrelated DNA. In the Pasteurellaceae and Neisseriaceae the molecular basis of this specificity is preferential binding of the uptake machinery to short DNA sequences present in thousands of copies in each species' genome. Such sequences are referred to as uptake signal sequences (USSs) in the Pasteurellaceae and DNA uptake sequences (DUSs) in the Neisseriaceae; they are not known in other naturally transformable bacteria $[7,8]$.

The best-characterized uptake sequences are those of Haemophilus influenzae and Neisseria meningitidis and N. gonorrhoeae. The preferred sequences themselves appear to have little in common: the core $H$. influenzae USS is 5'-AAGTGCGGT (5'-ACCGCACTT in the reverse orientation), with two AT-rich motifs on the 3' side of the standard orientation [9], whereas the Neisseria DUS is GCCGTCTGAA with no flanking motifs [10]. However, similarities in genomic frequencies and distributions suggest that they have arisen by similar processes. Both USSs and DUSs are present in their respective genomes at frequencies close to one copy per $\mathrm{kb}$ and both show no significant orientation bias. Both types are distributed somewhat more regularly around their genomes than expected for randomly located repeats, but both have some copies occurring in closely spaced oppositely oriented pairs [7]. Both USSs and DUSs are preferentially found in non-coding DNA sequences, but both have many copies in coding sequences. Both types are also overrepresented in the genomes of at least some other members of their genus or family $[4,7-9,11$ 13].

One puzzling attribute shared by USSs and DUSs is an unusually strong consensus, with each genome containing many more copies that perfectly match its consensus core sequence than singly mismatched copies. This pattern is typical of young transposons and other genetic elements that multiply by copying, but very different from the more relaxed consensus typical of sequences that function as binding sites for regulatory proteins, which arise by point mutation of pre-existing sequences. Because USSs are thought to function by binding to DNA-receptor proteins at the cell surface $[14,15]$, their very strong consensus is anomalous. The explanation might be that the DNA uptake machinery at the cell surface binds DNA with much higher specificity than do intracellular DNA-binding proteins. However the possibility that USSs arise by a copying process has not been excluded.

Previous analysis has found that copies of the $H$. influenzae USS are abundant in the genomes of several other members of the Pasteurellaceae (Actinobacillus actinomycetemcomitans, Pasteurella multocida, and H. somnus), and comparison of homologous genes in $H$. influenzae and $P$. multocida has shown that individual USSs can be stable over hundreds of millions of years [13]. However, preliminary examinations of the sequenced genomes of the Pasteurellaceans Mannheimia haemolytica (by Sarah Highlander) and Actinobacillus pleuropneumoniae (by ourselves) found that the $H$. influenzae USS was much less abundant than a related sequence that differs at several positions, suggesting that these genomes might contain a variant USS.

Insight into the evolution of competence will depend on an improved understanding of Pasteurellaceaen phylogeny. Almost all Pasteurellaceae (gamma-proteobacteria) are commensals and/or pathogens of the mucosal surfaces of vertebrates, primarily birds and mammals, and several are important human pathogens. Although phylogenetic analysis based on $16 \mathrm{~S}$ rRNA sequences has confirmed that the family is monophyletic, the relationships of its members remain poorly resolved. Two recently published phy- 

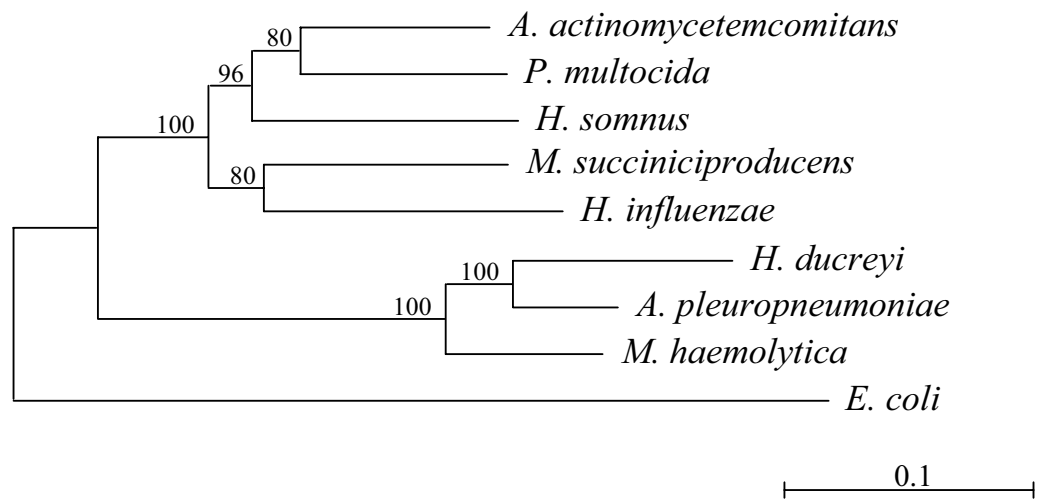

\section{Figure I}

Phylogeny of 8 Pasteurellacean species. Phylogenetic analysis was based on amino acid sequence of 12 protein-coding genes (listed in Methods) with homologues in all 8 Pasteurellacean genomes and in E. coli. The scale bar is 0.1 substitutions per site.

logenies used small-subunit rRNA sequences from 83 Pasteurellacean taxa [16] and partial sequences of the housekeeping genes atpD, infB and $r p o B$ from 28-36 strains [17], but the resolution was unsatisfactory, with many low bootstrap values and unresolved nodes.

Here we use the concatenated sequences of 12 proteins to construct a well-resolved phylogenetic tree for the species of Pasteurellaceae with genome sequences available. This tree then serves as a framework against which we characterize the long-term evolution of competence genes and of DNA uptake specificity.

\section{Results and Discussion A robust Pasteurellacean phylogeny}

The amino acid sequences of 12 well-conserved genes were identified from the available published and draft sequences of Pasteurellacean genomes and used to infer the consensus phylogeny shown in Figure 1. Homologous E. coli genes were used as the outgroup. The chosen genes did not contain the $H$. influenzae USS, were distributed around the $H$. influenzae genome, and had strong homologues in the other genomes. Intracellular proteins were chosen to preclude the diversifying selection that can bias evolution of proteins exposed on the cell surface, and genes with base compositions typical of their species' genomes were used to preclude recent horizontal transfer.

The resulting phylogenetic tree (Fig. 1) identified two primary subclades within the Pasteurellaceae. These are referred to below as the Hin subclade and the Apl subclade. The statistical support for these subclades is robust, with bootstrap values of $100 \%$. Independent phylogenies done for each protein separately all grouped A. pleuropneumoniae, M. haemolytica and $H$. ducreyi together as a distinct subclade from the five other species. The 100\% bootstrap values place A. pleuropneumoniae and $H$. ducreyi as sister species, but the branching order in the Hin subclade remains uncertain.

This phylogeny is restricted to the eight species with sequenced genomes, but it is the first Pasteurellacean tree to have strong statistical support. It differs in many respects from both the 16S rRNA and protein phylogenies previously published for the Pasteurellaceae. However in our view these discrepancies are the consequence of most branches of those earlier phylogenies having very poor bootstrap support, making them intrinsically unreliable, and so should not be a cause for concern. The Apl subclade it predicts was also supported by the protein tree of Christensen et al. [17]. Although the Apl subclade is not seen in Christensen et al.'s small-subunit rRNA tree, the resolution of that region of their tree is poor (best bootstraps are $69 \%$ and $75 \%$ ) [16]. The topology of an earlier tree based on small subunit rRNA sequences agrees with ours, although none of the relevant bootstrap values in that tree are significant [18]. The new tree also confirms what the previous more-detailed but less-well-supported trees had predicted - that within the Pasteurellaceae true evolutionary relatedness is not well correlated with many of the features previously used to assign isolates to genus $[17,19,20]$.

The genus assignment of M. succiniciproducens provides an example. This species, isolated from bovine rumen, was assigned to Mannheimia based on a simple small-subunit rRNA tree with no bootstrap analysis [21]. Our phylogenetic analysis instead places the two Mannheimia species in separate subclades. The $80 \%$ bootstrap score supporting M. succiniciproducens's placement as the sister group to 
Table I: Competence genes in Pasteurellacean genomes

\begin{tabular}{|c|c|c|c|c|c|c|c|c|c|}
\hline \multirow[t]{2}{*}{ Gene } & \multirow[t]{2}{*}{$\mathrm{HI \#}$} & \multicolumn{8}{|c|}{ Species } \\
\hline & & Hin & Aac & Pmu & Hso & Msu & $A p l$ & Mha & Hdu \\
\hline comA & 0439 & + & + & + & + & + & + & + & def \\
\hline $\operatorname{com} B$ & 0438 & + & + & + & + & + & + & + & def \\
\hline comC & 0437 & + & + & + & + & + & + & + & + \\
\hline comD & 0436 & + & + & + & def & + & + & + & + \\
\hline comE & 0435 & + & + & + & def & + & + & + & + \\
\hline $\mathrm{comF}$ & 0434 & + & + & + & + & + & + & + & + \\
\hline comEl & 1008 & + & + & + & + & + & + & + & + \\
\hline comM & 1117 & + & + & + & + & def & + & + & def \\
\hline dprA & 0985 & + & + & + & + & + & + & + & + \\
\hline pilA & 0299 & + & + & + & + & + & + & + & + \\
\hline pilB & 0298 & + & + & + & + & + & + & def & + \\
\hline pilc & 0297 & + & + & + & + & + & + & + & + \\
\hline pilD & 0296 & + & + & + & + & + & + & + & + \\
\hline rec2 & 0061 & + & + & + & + & + & + & + & + \\
\hline \multirow[t]{4}{*}{$s x y$} & 0601 & + & + & + & + & + & + & + & + \\
\hline & 0366 & + & + & + & + & + & + & + & + \\
\hline & 0938 & + & + & + & + & + & + & + & + \\
\hline & 0939 & + & + & + & + & + & + & + & + \\
\hline
\end{tabular}

+ Gene present

def Gene present but defective due to mutation

H. influenzae in Fig. 1 is too low to rule out a closer affinity with $P$. multocida. Hong et al. compared the M. succiniciproducens genome sequence to those of both $P$. multocida and $H$. influenzae; more genes are shared with the former, but the amino acid identities are higher with the latter $[22,23]$. In any case it is striking to find a rumen bacterium as such a close relative of bacteria otherwise restricted to respiratory mucosa.

\section{Competence genes in Pasteurellacean genomes}

Natural transformation has been demonstrated experimentally in only three of the eight sequenced species $(H$. influenzae, A. actinomycetemcomitans and A. pleuropneumoniae $[12,24,25])$. Only two other species within the Pasteurellaceae have also been shown to be naturally competent (Haemophilus parasuis [26] and Haemophilus parainfluenzae [4,27]). A number of other species have resisted multiple attempts at transformation in the laboratory, but their nontransformability could be misleading, as cellular processes important in the natural environment may not be induced under laboratory culture conditions.

VanWagoner et al. identified homologues of several $H$. influenzae competence genes (HI0366, HI0938 and HI0939) in most sequenced Pasteurellacean genomes [28]. As we have recently identified the complete competence regulon of $H$. influenzae, we examined the genomes of all of the sequenced Pasteurellaceae for homologues of all of these genes [29]. Table 1 shows that all of the genomes contain recognizable homologues of all of the genes known to be required for competence in $H$. influen$z a e$, as well as homologues of most other genes consistently occurring in the same operons.

However not all of the genes in this ancestor's sequenced descendants appear to be functional. The H. ducreyi comA, $\operatorname{com} B$ and $\operatorname{com} M$ genes are interrupted by an internal stop codon $(\operatorname{com} A)$ and frameshifts $(\operatorname{comB}$ and $\operatorname{com} M)$. A deletion in the $H$. somnus genome fuses the 5 ' portion of comD to the 3' $67 \%$ of comE, which also contains a frameshift. A $17 \mathrm{~kb}$ insertion disrupts the com $M$ gene of $M$. succiniciproducens, and most of pilB in $M$. haemolytica has been deleted. In some cases, examination of genome sequences from different isolates revealed discrepancies; these may result from strain-specific variation or from the preliminary nature of some of the sequences used. Only the sequenced genomes of $H$ influenzae, A. actinomycetemcomitans, P. multocida and A. pleuropneumoniae retain fully intact sets of competence genes.

What inferences can be drawn about the evolution of competence? First, the most parsimonious explanation for the presence of all competence genes in all genomes is that the ancestral Pasteurellacean had functional copies of all these genes and was naturally competent. When and where did this ancestor live? Although dating bacterial divergences is highly problematic, the most recent common ancestor of $H$. influenzae and P. multocida, and thus of the Hin-subclade in Fig. 1, has been estimated to have 


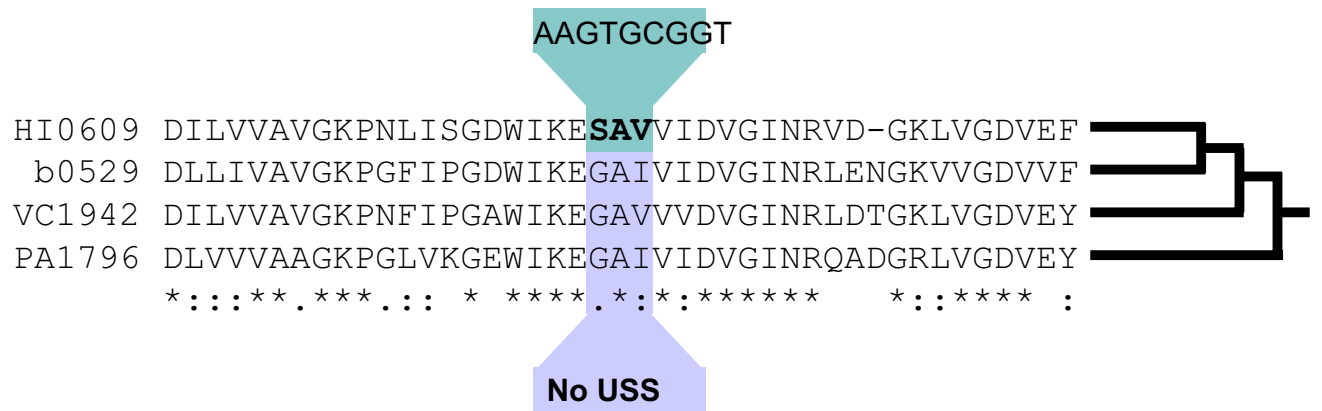

Figure 2

Example gap analysis of a USS-homologous peptide. A USS-encoded peptide and flanking region within the $H$. influenzae folD gene (HI0609; amino acids 203-245), aligned with folD proteins from: E.coli (b0529; amino acids 203-246), Vibrio cholerae (VCI942; amino acids 228-27I), and Pseudomonas aeruginosa (PAI796; amino acids 203-246). The sketch at the right shows the phylogenetic relationships of these taxa [57].

lived about 270 million years ago (mya), and last common ancestor of the entire family must be older still $[30,31]$. Thus the origin of the Pasteurellaceae is likely to have long predated the origin of mammals (c.195 mya) and may be contemporaneous with the origin of tetrapods about 360 mya. If so, it is possible that these bacteria moved into the respiratory tract and used the abundant DNA found there [32] almost as soon as the first respiratory tracts evolved.

What then explains the sporadic distribution of competence in its descendants? Three of the five genomes from 'non-transformable' species we analyzed carry obvious genetic defects that would prevent DNA uptake. (Loss of comM in M. haemolytica would only prevent transformation.) Each defect is unique and so must have arisen since the most recent divergence in its lineage. Furthermore, the substitution rate indicated by the scale bar on Fig. 1 allows estimation of the minimum number of chain-terminating and frameshift mutations expected to have accumulated since loss of a competence gene removed selection on other competence-specific genes. The scarcity of such mutations in each of these strains $(0,1$ or 2$)$ suggests that competence was lost quite recently. This is consistent also with the high densities and strong consensuses of the USS in all genomes except $H$. ducreyi. Frequent recent losses of competence would also explain the reported variation in competence within populations [3-6].

\section{Uptake signal sequences (USS) are not insertions}

One goal of this work was to use USS distribution to make inferences about the evolution of DNA uptake specificity. However, the anomalously strong consensuses of $H$. influenzae USSs (and other USSs) raised the concern that they might have been produced by insertion of a replicating element rather than by point mutations in pre-existing sequences. Fortunately the mode of USS origin makes a simple prediction about the positions of gaps in sequence alignments. If individual $H$. influenzae USSs have arisen by insertion, gaps should be seen when the segments containing these USS are aligned with homologous sequences from genomes that diverged before USS arose. We used this prediction to test whether the many $H$. influenzae USSs in protein coding sequences arose in an ancestral Pasteurellacean by insertion or by accumulation of point mutations in the ancestral genes.

Because of the evolutionary distance between $H$. influen$z a e$ and species with no USS, the alignments were done between predicted amino acid sequences rather than nucleotide sequences. Segments of well-conserved $H$. influenzae proteins, centred on USS-encoded amino acids, were aligned with homologous protein segments from Escherichia coli, Vibrio cholerae and Pseudomonas aeruginosa, whose genomes do not contain USS-like repeats. A typical alignment is shown in Fig 2, along with a sketch of the evolutionary relationships of these bacteria.

Of the $956 \mathrm{H}$. influenzae USS in protein-coding regions ( $65 \%$ of all USS), 158 were in protein segments that could be aligned with $=50 \%$ identity to homologues from all three other species. Only 24\% (115/474) of the individual E. coli, $V$. cholerae and $P$. aeruginosa proteins alignments contained gaps, and most gaps were outside of the segment encoded by the USS core. Similar results were seen in alignments between homologues from outside the gamma-proteobacteria and the subset of $H$. influenzae protein sequences sufficiently well conserved to align well (results not shown). The homology between amino acids that are USS-encoded in $H$. influenzae and amino acids in 
Table 2: Most common 8-, 9- and 10-mers in Pasteurellacean genome sequences

\begin{tabular}{|c|c|c|c|c|c|}
\hline Genome $^{a}$ & $\% G+C$ & Size $(\mathrm{Mb})$ & $\begin{array}{l}\text { Most common } 10-\text { merb }^{\mathrm{b}} \text { (number, } \\
\text { fold over-rep) }\end{array}$ & $\begin{array}{l}\text { Most common 9-merb (number, fold } \\
\text { over-rep) }\end{array}$ & $\begin{array}{l}\text { Most common 8-merb (number, fold } \\
\text { over-rep) }\end{array}$ \\
\hline Hin & 38.1 & 1.8 & AAAGTGCGGT $(1115,429 X)$ & AAGTGCGGT (I47I, I75X) & AAGTGCGG $(1687,63 X)$ \\
\hline Aac & 44.4 & 2.1 & AAAGTGCGGT $(1422,384 X)$ & AAGTGCGGT $(1760,132 X)$ & AAGTGCGG $(1863,39 X)$ \\
\hline Pmu & 40.4 & 2.3 & AAAGTGCGGT $(700,200 X)$ & AAGTGCGGT $(927,79 X)$ & AAGTGCGG $(1013,26 X)$ \\
\hline Hso & 37 & 2.1 & AAAGTGCGGT $(776,273 X)$ & AAGTGCGGT $(1216,135 X)$ & AAGTGCGG $(1446,5 I X)$ \\
\hline Msu & 42.5 & 2.3 & AAAGTGCGGT $(1297,333 X)$ & AAGTGCGGT $(1485,1 \mathrm{I}$ IX) & AAGTGCGG $(1622,46 X)$ \\
\hline$A p l$ & 41.4 & 2.2 & ACAAGCGGTC $(429,187 X)$ & ACAAGCGGT $(742,68 X)$ & CAAGCGGT $(1361,36 X)$ \\
\hline Mha & 41 & 2.7 & ACAAGCGGTC $(506,181 X)$ & ACAAGCGGT $(973,70 X)$ & CAAGCGGT $(1636,48 X)$ \\
\hline$H d u^{c}$ & 38.2 & 1.76 & $\begin{array}{l}\text { TTTTGCAAAA }(106,9.6 \mathrm{X}) \\
\text { AATAAGCGGTc }(95,21 \mathrm{X}) \\
\text { AACAAGCGGTc }(85,31 \mathrm{X})\end{array}$ & $\begin{array}{l}\text { AATAAAAAA }(25 I, 2.8 X) \\
\text { ACAAGCGGTc }(199,23 X)\end{array}$ & $\begin{array}{l}\text { AAAAATAA }(680,2.3 X) \\
\text { CAAGCGGTc }(464,16 X)\end{array}$ \\
\hline
\end{tabular}

\footnotetext{
a Hin: H. influenzae; Aac: A.s actinomycetemcomitans; Pmu: P. multocida; Hso: H. somnus; Msu: M. succiniciproducens; Apl: A. pleuropneumoniae; Mha: M. haemolytica; Hdu: H. ducreyi.

$\mathrm{b}$ The first number in parentheses is the combined number of copies of the sequence and its reverse complement. The second number in parentheses is the fold over-representation of each repeat compared to a random-sequence genome of the same size and base composition.

c The additional entries for $H$. ducreyi are the copy numbers of the most frequent USS-like repeats.
}

distant relatives confirms that USSs have arisen by nucleotide substitutions in pre-existing sequences and not by insertions of a replicative element.

\section{All Pasteurellacean genomes contain USS-like repeats}

The next step was to characterize the phylogenetic distribution of USS. Bakkali et al. found that the only overrepresented short repeats in the $P$. multocida genome are variants of the $9 \mathrm{bp} \mathrm{H}$. influenzae USS core [13]. They also found the $H$. influenzae USS core to be highly overrepresented in the $H$. somnus and $A$. actinomycetemcomitans genomes but did not survey other repeats. To avoid the bias of searching for a specific USS sequence, we extended this analysis by counting all 6-12 bp repeats in all eight genomes (Table 2) and calculating the number of each repeat expected in a random-sequence genome of the same size and base composition.

Table 2 shows that all genomes had highly overrepresented repeats related to the $H$. influenzae USS. The most common 9-mer repeats in the genomes of A. actinomycetemcomitans, P. multocida, M. succiniciproducens and $H$. somnus are the $H$. influenzae USS core AAGTGCGGT and its reverse complement. All of the ten most abundant 8mer, 9-mer and 10-mer repeats in these genomes also contain or closely overlap this 9-mer. We will refer to this as the Hin-type USS. However the most frequent 9-mer repeats in the genomes of A. pleuropneumoniae and $M$. haemolytica differed from the Hin-type USS at the second, third and fourth positions (ACAAGCGGT rather than AAGTGCGGT); we will refer to this as the Apl-type USS. The most abundant repeats in the $H$. ducreyi genome were not recognizable USSs but simple palindromes and strings of As and Ts, so Table 2 also gives the frequencies of the most common USS-like 8,9 and 10-mers for this genome. These resembled the Apl-type USS but their copy numbers were substantially lower than in the other genomes. (Although the 10-mer AATAAGCGGT was the most common USS-like 10-mer repeat, ATAAGCGGT and TAAGCGGT were not among the 50 most frequent 9-mers and 8-mers.)

Each genome was specifically checked for repeats of the other USS type. The frequencies of both types of $9 \mathrm{bp}$ USSs per $\mathrm{Mb}$ sequence in all eight genomes are shown in Fig. 3A. Only 4 copies per Mb would be expected in randomsequence genomes of the same base compositions. Although the minority USS type (e.g. Hin-type USS in A. pleuropneumoniae) is several-fold overrepresented in each genome, it is not significantly more frequent than other 9mers sharing the global consensus ANNNGCGGT. Thus each genome appears to have a predominant subcladespecific USS type.

Fig. 3B shows, for each Pasteurellacean genome, the ratio of repeats perfectly matching each USS type to repeats with single mismatches to that type. Genomes with Hintype USSs resemble $H$. influenzae in having more perfect than singly mismatched copies, despite the 27 -fold greater number of possible sequences. The discrepancy is also seen for genomes with Apl-type USSs; with the exception of $H$. ducreyi, the ratio is substantially higher for the subclade-specific USS type than for the other type. The consistency of the pattern suggests that USS accumulation is shaped by similar forces in the different genomes.

\section{Detailed comparisons of USSs}

As USSs are thought to function by binding to DNA receptors on the cell surface, bases at different positions in the USS core would be expected to show consensus strengths reflecting their differing contributions to this DNA-protein binding. Sequence logos were used to visualize the 
representation of each base at each position of the USS (Figs. 4 and 5) [33]. In these logos the relative heights of the $A, G, C$ and $T$ in each stack shows the frequencies of the bases at that position, and the overall height of each stack of letters reflects the strength of the consensus at that position (the information content). The height of the stack is especially sensitive to minor changes in the frequency of a very frequent base (e.g. if the frequency of the most common base falls from 1.0 to 0.9 the height falls from 2.0 to 1.6$)$.

Fig. 4A shows the differences in consensus strengths at the USS core positions. In all genomes with Hin-type USSs most core positions appear to have roughly equal consensus strengths, suggesting that all make similar contributions to binding specificity. The exceptions are the final $\mathrm{T}$, which has a weaker consensus in $H$. influenzae and $H$. somnus, and the first $\mathrm{G}$ in M. succiniproducens. For genomes with the Apl-type USS the first two positions of USS cores show a weaker consensus than the other positions, suggesting that they may make a lesser contribution to the specificity of DNA binding and uptake. Confirmation of these predictions must await identification and characterization of the proteins or other molecules that interact with the USS at the cell surface.

The H. influenzae and A. actinomycetemcomitans USSs have been shown to also share conserved motifs (segments 2 and 3 ) on the 3 ' side of the USS core $[9,12]$. The importance of segment 2 was experimentally demonstrated by Danner and coworkers, who showed that USS-containing DNA fragments ethylated at bases in this region were not taken up by competent $H$. influenzae cells [15]. The functions of these positions in DNA uptake are not known; they may be additional sites of contact with the DNA receptor, or they may be involved in DNA bending or kinking during uptake.

Fig. 4B shows the consensuses of positions flanking the core USSs in each of the eight genomes. As viewed in the standard orientation, all USS consensuses have an AT-rich motif just $3^{\prime}$ of the core (segment 2; positions 22-27 as numbered in Fig. 4B), with the first bases usually 'A's and the final 3 or 4 bases 'T's. A second AT-rich motif is seen further downstream (segment 3). In the Hin-type USS this 6 nt-motif segment consists primarily of Ts, and is centred 12 positions to the right of segment 2. In A. pleuropneumoniae and $M$. haemolytica, segment 3 extends slightly farther to the left and substantially farther to the right, and has the more complex consensus AAAATTTTGCAAAT. Although the H. ducreyi USS consensus in segments 2 and 3 resembles the Apl-type motifs, it is much weaker. Together with the lower frequency of USS in its genome, and the presence of inactivating mutations in three of its competence genes, this suggests a relatively ancient loss of ability to take up DNA.

The consensuses in segments 2 and 3 of the A. pleuropneumoniae and $M$. haemolytica USSs were particularly strong and extensive. To compare their strengths to that of the core USS we repeated the above analysis in reverse. We chose the nine bases making the strongest contribution to segment 2 and segment 3 (ATTTNNNNNNNNNTTTGC) or to segment 3 alone (TTTTGCAAA) and identified and aligned all A. pleuropneumoniae genomic segments containing them (560 and 454 segments respectively). The resulting logos (Fig. 5A and $5 \mathrm{~B}$ ) show that many of the segments bearing these motifs also contained all but the first two bases of the Apl-type USS core. The weak correlation of the first two positions of the core with the flanking segments may mean that these positions play a lesser role in USS function than the rest of the core, with its larger segment 3 making a greater contribution to the binding specificity. A logo using only the 164 sequences with 12 matches to segments 2 and 3 was even more effective, recovering the full core consensus (Fig. 5C). Taken together, these analyses suggest that, at least in A. pleuropneumoniae, the motifs in segments 2 and 3 may be as important for DNA uptake as the USS core.

\section{USS in $\boldsymbol{H}$. parasuis}

A recent paper reported that $H$. parasuis has the core USS GAGTTCGGT, which differs from both the Hin and Apl types[26]. However this conclusion was based on analysis of a single putative USS in a cloned $413 \mathrm{bp}$ fragment. We have examined all the available $H$. parasuis sequences $(86,701 \mathrm{nt}$, mainly in ORFs) and find, in addition to the one copy of this repeat described by Bigas et al., four copies of the Hin-type USS, fourteen copies of the Apl-type USS, and seventeen copies of sequences differing at single positions from the Apl-type USS. This suggests that $H$. parasuis has an Apl-type USS, which would be consistent both with previous phylogenetic analysis placing it in a strongly supported subclade with $M$. haemolytica and $A$. pleuropneumoniae $[17,34]$ and with the ability of $A$. pleuropneumoniae DNA to efficiently transform $H$. parasuis [35].

\section{$H$. influenzae and $A$. pleuropneumoniae recognize subclade-specific USSs}

A. actinomycetemcomitans (Hin-type USS) has already been shown to preferentially take up its own and $H$. influenzae DNAs [12], but for most of the other species the role of the putative USS in DNA uptake could not be directly tested because no competent isolate has been identified. However A. pleuropneumoniae strain HS143 (serotype 15) has recently been shown to be much more competent than other strains (J. Bossé, manuscript in preparation), allowing us to test its uptake specificity by three different exper- 

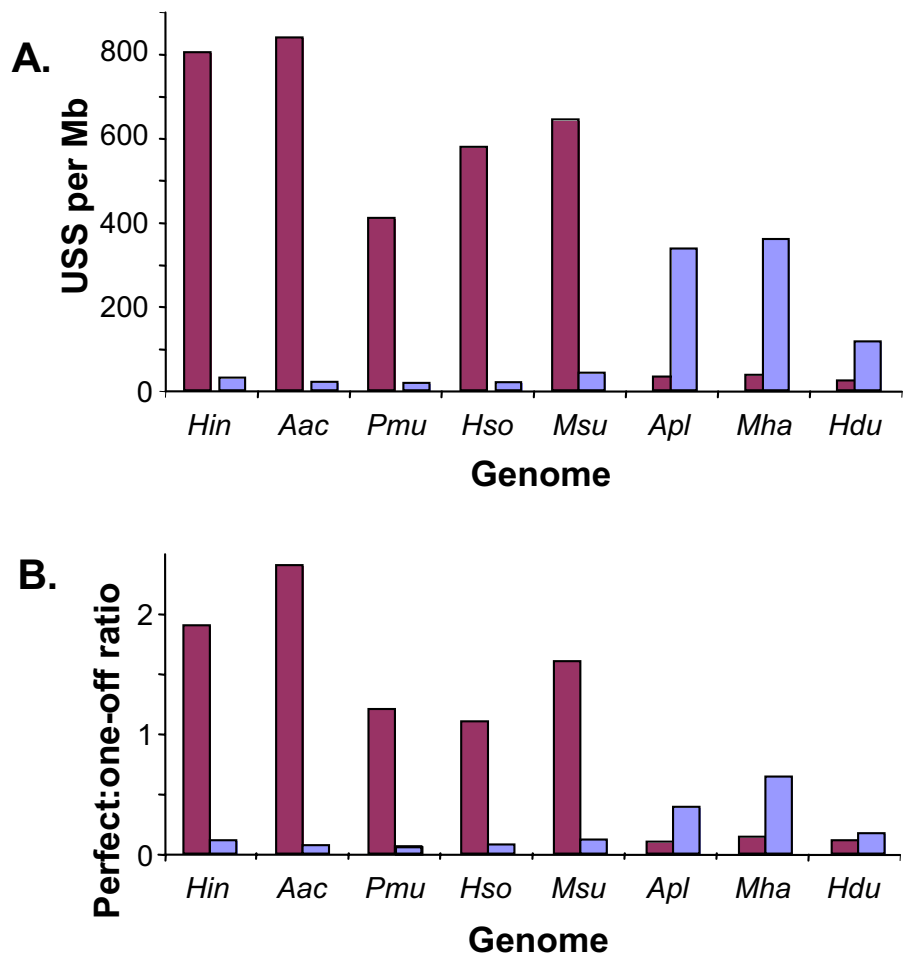

\section{Figure 3}

USS frequencies in sequenced Pasteurellacean genomes. Red: Hin-type USSs (AAGTGCGGT); blue: Apl-type USSs (ACAAGCGGT). A. Frequencies of 9 bp core USSs of each type per Mb of genome. B. Ratios of perfect to singly mismatched 9 bp USS cores.

iments. Each confirmed that competent $A$. pleuropneumoniae cells preferentially take up DNA fragments containing the Apl-type USS.

The solid bars in Figure 6A and 6B show measurements of uptake by competent $H$. influenzae and A. pleuropneumoniae cells of radiolabelled 220 bp DNA fragments containing synthetic $H$. influenzae and A. pleuropneumoniae USSs. These USSs were designed to contain the most common base at each position of the extended USSs described above; a control fragment contained a randomized version of the $H$. influenzae USS sequence. As expected, $H$. influenzae took up about 1500-fold more DNA containing its USS than control DNA (Fig. 6A; note the log scale). The function of the Apl-type putative USS was confirmed; A. pleuropneumoniae took up about 17 -fold more DNA with its USS than control DNA (Fig. 6B). Each species also took up substantially less DNA containing the heterologous USS type than its own type (only about twice as much as control DNA), confirming that the DNA uptake machinery discriminates between the two types.

Uptake of chromosomal DNA may provide a more biologically relevant measure of specificity. The dashed bars in Fig. 6A and 6B show uptake of radiolabelled chromosomal DNAs by competent H. influenzae and A. pleuropneumoniae cells. In this assay $H$. influenzae took up 50-fold more H. influenzae DNA than the control E. coli DNA (Fig. 6A), and A. pleuropneumoniae took up about 37-fold more A. pleuropneumoniae DNA than E. coli DNA (Fig. 6B). In both chromosomal and synthetic-USS uptake experiments A. pleuropneumoniae took up substantially less DNA than did $H$. influenzae, consistent with its lower transformation frequency.

Consideration of the relative densities of the two USS types in the three genomes (H. influenzae: A. pleuropneumoniae: E. coli; 198:8:1 (Hin-type USSs) and 4.4:48:1 (Apltype USSs)) allows clarification of the degree to which each species takes up DNA of the other type. First, the high density of Hin-type USS in the $H$. influenzae genome means that most chromosomal DNA fragments $(\geq 50 \mathrm{~kb}$ long) would have contained at least several USS, reducing the contribution of each USS to uptake. Second, the presence of heterologous USS in the two Pasteurellacean genomes can explain $H$. influenzae's 6-fold higher uptake of A. pleuropneumoniae DNA than E. coli DNA, but is likely insufficient to explain A. pleuropneumoniae's 32-fold 

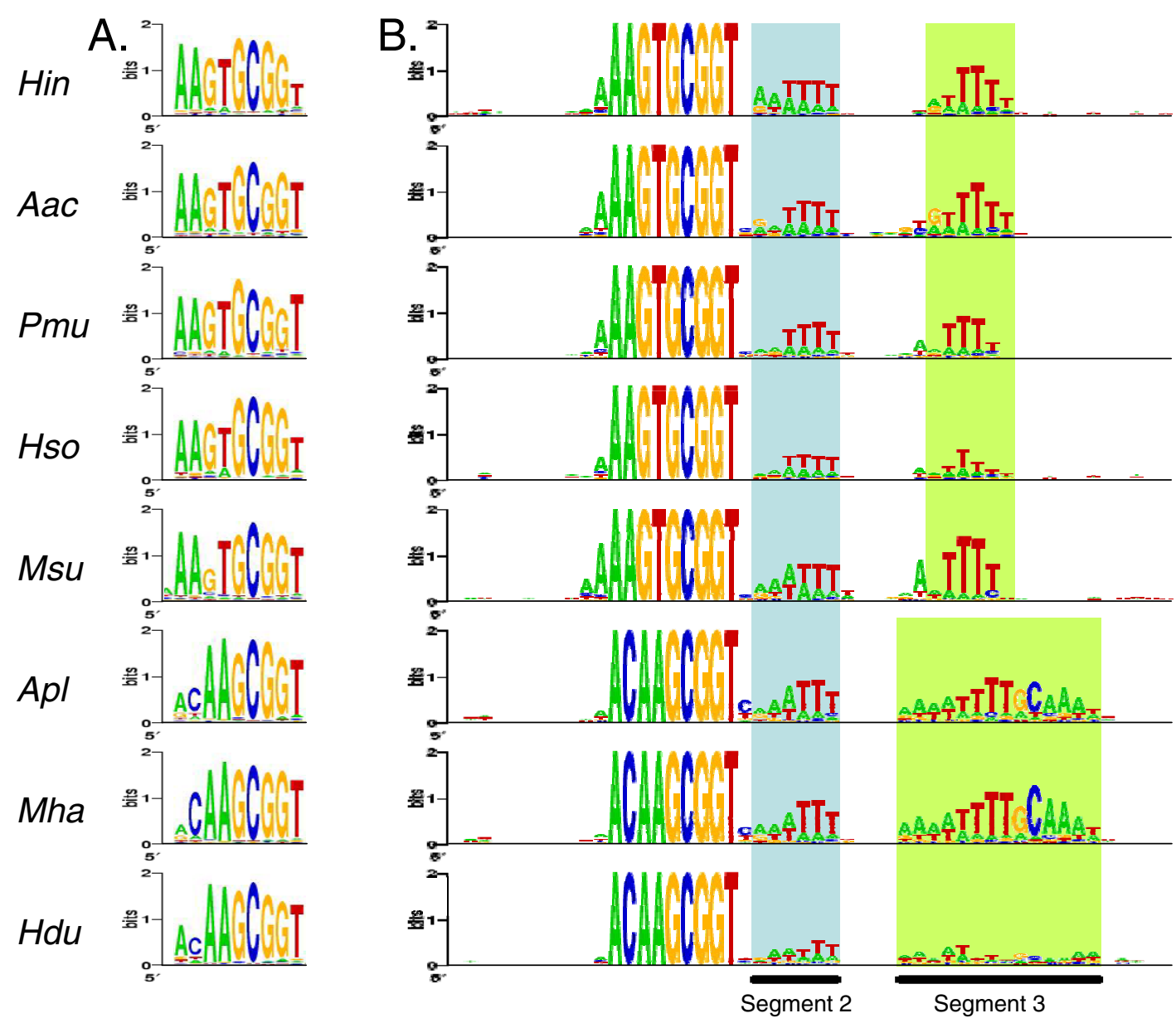

Figure 4

WebLogos for USSs and surrounding sequence in 8 genomes. A. Logos based on 9 bp segments with perfect or oneoff matches to the 9 bp USS. B. Logos based on 50 bp segments with perfect matches to the 9 bp USS.

higher uptake of $H$. influenzae DNA than E. coli DNA. These results suggest that the A. pleuropneumoniae uptake machinery does indeed weakly recognize the Hin-type USS, and do not preclude a similar overlap in specificity by the $H$. influenzae uptake machinery.

Figure 7 shows the extent to which cells preferentially take up genetically marked conspecific DNA in the presence of competing DNA from their own strain or another species. This is a more sensitive measure of uptake bias than the uptake of pure DNAs tested above. Fig. 7A shows the results of uptake-competition assays using $H$. influenzae cells and a constant amount of $H$. influenzae chromosomal DNA carrying a novobiocin resistance allele. As expected, unmarked $H$. influenzae DNA competed strongly but B. subtilis DNA, which does not contain overrepresented USS-like repeats, did not [36]. A. pleuropneumoniae DNA did not compete for uptake. Fig. 7B shows that A. pleuropneumoniae took up its own DNA in prefer- ence to both $H$. influenzae DNA and B. subtilis DNA. These results confirm that the DNA uptake machineries of both $H$. influenzae and A. pleuropneumoniae discriminate strongly in favour of DNAs containing their own USS type. $H$. parasuis DNA was also tested; it did not compete for uptake by $H$. influenzae (Fig. 7A), but competed with A. pleuropneumoniae DNA for uptake by A. pleuropneumoniae to an extent consistent with the density of Apl-type USS in its DNA.

We did not test whether cells could discriminate between DNAs from species in the same subclade. However, as an earlier measure of relatedness among the Pasteurellaceae, Albritton et al. examined the ability of DNAs from various species to compete with $H$. influenzae DNA for uptake by competent $H$. influenzae cells [37]. The ability to compete for uptake correctly predicted the USS distributions we have found: DNAs from A. actinomycetemcomitans and $P$. multocida (Hin subclade) competed strongly (54\% and 


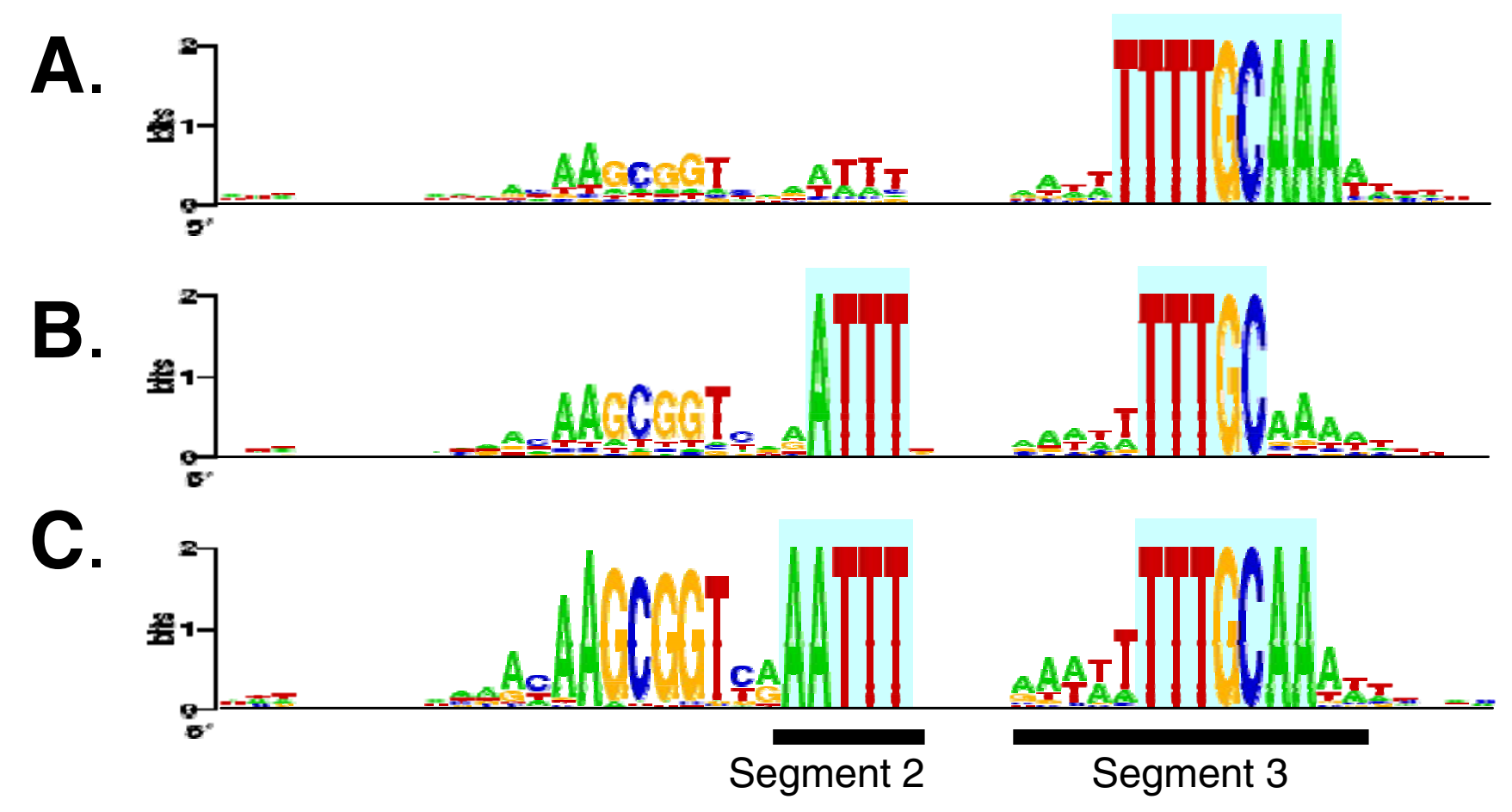

Figure 5

WebLogos for 50 bp segments of the A. pleuropneumoniae genome. The highlighting indicates the positions used to choose sequences for analysis.A. Logos for segments containing the motif TTTTGCAAA. B. Logos for segments containing the motif ATTTNNNNNNNNNTTTGC. C. Logos for segments containing the motif AATTTNNNNNNNNNTTTGCAA.

$44 \%$ as well as $H$. influenzae DNA), but DNA from A. pleuropneumoniae (Apl subclade) competed only poorly (7\%). Although the other sequenced species were not tested, the competition shown by DNA of non-sequenced Pasteurellacean species is likely to predict the USS types they contain. Thus the strong competition Albritton et al. observed by $H$. parainfluenzae, H. aphrophilus, H. paraphrophilus and P. pneumotropica DNAs suggests that they likely carry Hintype USSs. Consistent with this, $H$. parainfluenzae is known to have Hin-type USSs [15], and Christensen et al.'s rRNA and protein trees place $P$. pneumotropica close to $P$. multocida with reasonable bootstrap support; their rRNA tree also places $H$. aphrophilus and $H$. paraphrophilus close to $H$. influenzae with modest bootstrap support. In contrast, Albritton et al. found that DNAs of P. ureae, A. lignieresii and A. equuli competed very poorly with $H$. influenzae DNA. These taxa are closely linked to A. pleuropneumoniae in Christensen et al.'s rRNA tree, and A. lignieresi is the sister taxon to A. pleuropneumoniae in their protein trees, supporting the hypothesis that they have Apl-type USSs [16,17].

The shared features of the Pasteurellacean USS types may reflect generalized features of the DNA uptake process. The 9 bp USS cores may match the size of the recognition domain of the as-yet-unidentified DNA receptor protein, and are similar in length to the $10 \mathrm{bp}$ Neisseria core. The conservation of segment 2 and segment 3 in the Pasteurellaceae is intriguing, as conserved flanking motifs are not seen in Neisseria. It may be significant that the spacings between the USS core, segment 2 and segment 3 correspond roughly to single turns of helical DNA. We know it cannot be the case that $H$. influenzae cells initiate uptake by threading a DNA end through a membrane pore, because they efficiently take up covalently closed plasmids [38]. However DNA molecules are too highly charged and too stiff (persistence length about $50 \mathrm{~nm}$ or $150 \mathrm{bp}$ ) to simply pass sideways through the outer membrane. Together the USS core plus flanking motifs may allow the DNA to be sharply kinked (perhaps by strand separation), presenting a compact cross-section for membrane transit. Detailed understanding of the function of USSs will require more complete experimental studies of binding and incorporation of target DNA sequences.

\section{Conclusion}

The eight Pasteurellacean species we analyzed fall into two robust subclades. The genomes of all these bacteria contain homologues of all the $H$. influenzae genes known to be needed for DNA uptake, some of which have 

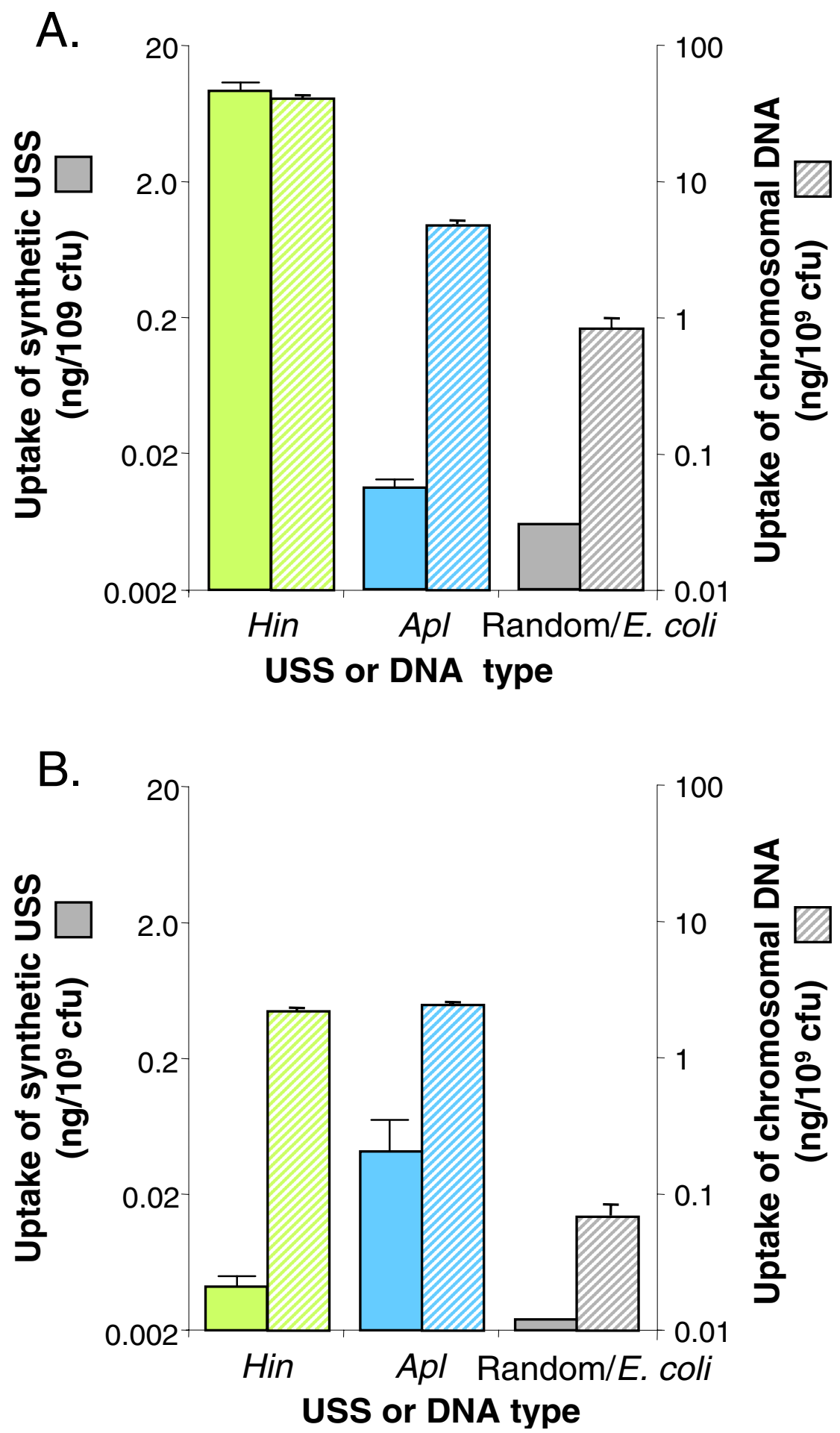

Figure 6

Uptake of synthetic USSs or chromosomal DNAs. The solid bars show uptake of 220 bp PCR fragments containing synthetic USS with the consensus sequences of $H$. influenzae (Hin), A. pleuropneumoniae (Apl) or randomized $H$. influenzae USS types. The dashed bars show uptake of chromosomal DNAs from $H$. influenzae (Hin), A. pleuropneumoniae (Apl) or E. coli. Error bars show the standard deviations of 3 replicate experiments, except for A. pleuropnemoniae in $\mathbf{A}$, which is from 4 replicate experiments. A. Uptake by $H$. influenzae. B. Uptake by A. pleuropneumoniae. 


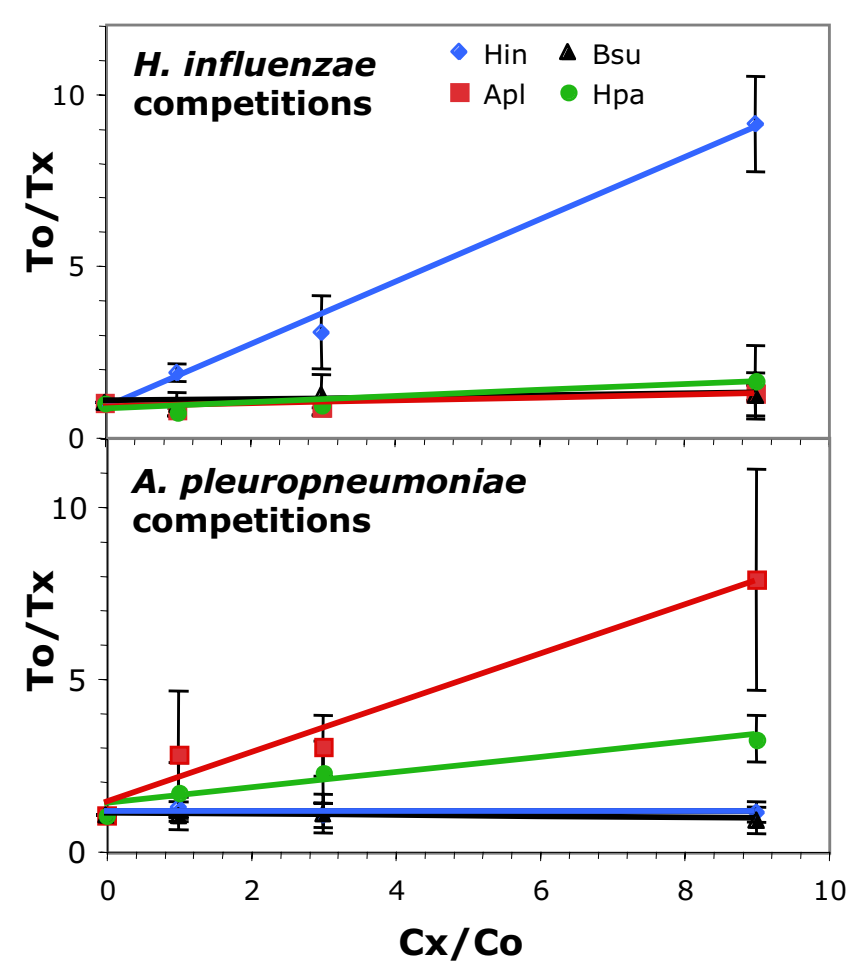

Figure 7

Double-reciprocal plots of uptake competition assays. Double-reciprocal plots of uptake competition assays. Cx/Co: ratio of competing DNA to genetically marked self DNA. To/Tx: ratio of number of transformants in the presence and absence of competing DNA Competing DNAs: blue diamonds, $H$. influenzae; red squares, $A$. pleuropneumoniae; black triangles, $B$. subtilis; green circles, $H$. parasuis. A. Competition in $H$. influenzae. B. Competition in A. pleuropneumoniae.

recently been inactivated by mutation. All of these genomes also contain high densities of genetically stable repeats, either the well-characterized $H$. influenzae USS or a related sequence, in each case comprising a 9 bp core and two adjacent AT-rich segments. The distribution of the Hin-type and Apl-type USSs corresponds to the two Pasteurellacean subclades. Competent members of these subclades discriminate between the two USS types, each preferring to take up DNA containing the USS typical of its own genome.

Taken together, these findings are consistent with the following model of the evolution of competence in the Pasteurellaceae: The ancestor of the sequenced Pasteurellaceae possessed a complete set of functional competence genes and was naturally competent, taking up DNA by a mechanism very similar to that used by $H$. influenzae today. The ancestral genome contained many USSs; these may or may not have been simpler than the USSs in its descendants, but likely had the common motif ANNNGCGGT in the USS core and included the AT-rich segment 2 and much of segment 3. During the initial diversification of the Pasteurellacean subclades the uptake specificity and USS consensus changed in parallel in one or both lineages. This divergence of genomic USSs may have been effectively complete before the divergence of the sequenced species within each subclade, with USS specificities remaining stable since then, although the existence of Pasteurellaceae with other diverged uptake specificities cannot be ruled out. Because the USS consensuses within each subclade are so similar, USS specificity will not enable competent bacteria to distinguish between DNAs derived from different species within their subclade. Because many of these DNAs are otherwise sufficiently diverged that recombination is not only inefficient but toxic $[37,39]$, forces other than exclusion of non-self DNA may be responsible for uptake specificity.

What are the implications for other bacterial families? We suggest that the evolutionary history of competence often follows the pattern shown in Fig. 8. In this model, the ancestors of many bacterial families were naturally competent but competence has been and continues to be frequently lost. Mutations causing loss of competence have not always been strongly selected against, and sometimes may have been actively favoured, so non-competent lineages often persist. However, over the long term the noncompetent lineages are selected against, so that all extant bacteria have recent ancestors who were competent. This hypothesis is consistent not only with our family-level analysis but with the extensive evidence of sporadic distribution of competence within individual species [3-6]. The pattern is similar to that seen for the mismatch repair system, where mutants with defects in mutation prevention can experience a short-term advantage but are eventually eliminated by selection against accumulating deleterious mutations [40].

Many questions remain unanswered. How deep is the ancestry of competence? Are some bacterial families ancestrally not competent? Have some modern species completely lost competence? Do genes introduced by conjugation or transduction ever restore competence to noncompetent lineages? Thanks to the ever-increasing availability of new genome sequences, answers to these questions will soon be within reach.

\section{Methods}

\section{Genome and gene sequences}

Choice of sequences

Complete and annotated genome sequences were available through NCBI for a single isolate each of P. multocida, M. succiniciproducens and H. ducreyi $[30,22,23]$. Sequences of four $H$. influenzae isolates were available [41]; we used the fully assembled and annotated sequence of the $\mathrm{Rd}$ 


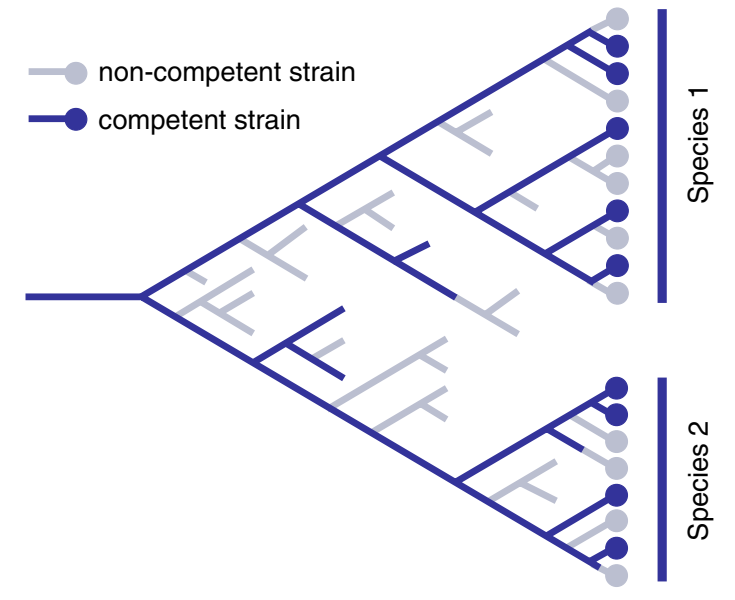

Figure 8

Model for the evolution of competence.

strain [42]. Sequencing is in progress for two $H$. somnus isolates, we used the more complete 129-PT sequence. Four incomplete $A$. pleuropneumoniae genome sequences were listed at NCBI; however we used our nearly complete sequence of strain L20 (serotype $5 \mathrm{~b}$, sequence available from JN on request). The genome sequences of $M$. haemolytica and A. actinomycetemcomitans are also in progress and not yet available at NCBI.

Complete genome sequences with annotations were retrieved from the NCBI website [43] for $H$. influenzae $\mathrm{Rd}$ KW20 (NC_000907.gbk), P. multocida subsp. multocida str. Pm70 (NC_002663.gbk), M. succiniciproducens MBEL55E (NC_006300.gbk), H. ducreyi 35000 HP (NC_002940.gbk), E. coli K12 (NC_000913.gbk), V. cholerae N16961 (NC_002505.gbk and NC002506.gbk) and $P$. aeruginosa PA01(NC_002516.gbk). Unfinished genome sequences were obtained for A. actinomycetemcomitans from [44] and for $H$. somnus 129-PT from [45]. Preliminary sequence data for $M$. haemolytica were obtained from the Baylor College of Medicine Human Genome Sequencing Center $[46,47]$. Open reading frames for genome sequences lacking annotation were identified from the draft sequence using the GLIMMER software package (now available at [48]).

\section{Phylogenetic analysis}

Phylogenetic analysis used the amino acid sequences of the following $12 \mathrm{H}$. influenzae genes and their best homologues in the other 7 Pasteurellaceaen genomes and in E. coli: gapdH (HI0001), lepA (HI0016), ffh (HI0106), serS (HI0110), secD (HI0240), dapA (HI0255), ruvB (HI0312), xerC (HI0676), ispB (HI0881), secA (HI0909), crp (HI0957), and dnaJ (HI1238). Homologues were identified using the BLASTP program $\left(\right.$ E-val $\left.<10^{-50}\right)$ in the BLAST package [49]. For each gene, the amino acid sequences from the 9 genomes were aligned using CLUSTALW with output in PHYLIP format [50]. The aligned sequences were inspected, the ends were trimmed to remove sequence missing in any of the 9 genomes, and the alignments of the 12 genes were concatenated to produce a single long alignment.

Phylogenetic analysis of the concatenated alignment used the PHYLIP software package [51]. ProML analysis using maximum likelihood with the JTT method and a gammaplus-invariant-sites distribution of rates across sites yielded a predicted tree with estimated phylogenetic distances. SeqBoot was then used to produce 100 datasets by bootstrapping resampling; these were put into ProML to generate phylogenetic trees. The final bootstrap analysis was done using the program Consense and the bootstrap values were added to the tree generated with the complete sequence above.

\section{Homology of USS-encoded peptides}

BLAST searches were used to identify those USS-containing $H$. influenzae genes that had homologues in all of $E$. coli, V. cholerae and P. aeruginosa. ClustalW was used to align the homologous protein sequences, with the default penalties of 10 for gap opening and 0.2 for gap extension. Analysis was restricted to 43aa segments centred on amino acids encoded by the USS core that showed $>50 \%$ amino acid identity across all homologues. All gaps within these alignments were tabulated.

\section{Repeat analysis}

The Perl program repeat_finder was developed to search genome sequences for abundant short DNA sequences (code available at [52]). It was used to tabulate the occurrences of the 20 most abundant 6-, 7-, 8-, 9-, 10-, 11-, and 12-mers for each of the 8 Pasteurellacaean genomes, along with the number of each expected for a randomsequence genome of that size and nucleotide composition.

All occurrences of the 9 bp putative USS core for each species were identified, and 50-bp sequence segments containing the core plus 11 bases upstream and 30 bases downstream were aligned. The program WebLogo $[7,53])$ was used to visualize the consensus for each USS. Similar analyses were done for each genome using all singly mismatched occurrences of the 9 bp core, and for A. pleuropneumoniae using consensus sequences derived from the two flanking regions.

\section{Bacterial strains and culture conditions}

A. pleuropneumoniae serotype 15 (strain HS143) and $H$. influenzae Rd (strain KW20) were grown in Brain Heart Infusion broth (Difco) supplemented with the recommended concentrations of NAD and hemin (H. influenzae 
only), and were made competent by transfer of exponentially growing cells to MIV starvation medium as described for $H$. influenzae [54]. Aliquots of competent cells were stored at $-80^{\circ} \mathrm{C}$ and thawed immediately before use.

\section{DNA labeling}

Chromosomal DNAs of H. influenzae Rd and A. pleuropneumoniae were labeled by nick-translation with alpha${ }^{33} \mathrm{P}$-dATP to specific activities of $2 \times 10^{7} \mathrm{cpm} / \mu \mathrm{g}$. Fragments of about $200 \mathrm{bp}$ centered synthetic USSs (USS-Hin: CCCAAAGTGCGGTTAATTTTTTACAGTATTTTTGGGTTCGAAAT; USS-Apl: GGAAACAAGCGGTCAAATTTGCCGAAAATTTTGCAAATTGGTACCT; USS-Ran: TCTTGTTAGAATCTGAGTGTTATTTAAAT) were PCRamplified from clones in pGEM [55] using primers with BglII ends. The amplified fragments were cut with BglII and end-labeled with Klenow polymerase using alpha${ }^{33} \mathrm{P}$-dATP, to specific activities of $10^{6}-10^{7} \mathrm{cpm} / \mu \mathrm{g}$.

\section{DNA uptake}

Competent cells of H. influenzae strain KW20 and A. pleuropneumoniae strain HS143 $\left(1.0 \mathrm{ml} ; \sim 1 \times 10^{9} \mathrm{cfu}\right)$ were incubated with $150 \mathrm{ng}$ of labeled chromosomal DNA or 20 ng of labeled PCR fragment for 15 minutes at $37^{\circ} \mathrm{C}$, followed by 5 minutes incubation with DNase I at $1 \mu \mathrm{g} /$ $\mathrm{ml}$. Cells were then washed three times at room temperature by pelleting and resuspension in $1.0 \mathrm{ml}$ of MIV, and the radioactivity of the pellets was counted.

\section{Transformation-competition experiments}

Competent cells of H. influenzae strain KW20 and A. pleuropneumoniae strain HS143 (0.2 ml) were incubated for 15 minutes at $37^{\circ} \mathrm{C}$ with $100 \mathrm{ng}$ of genetically marked conspecific DNA (MAP7 DNA for H. influenzae [54] and sodC::Kan DNA for A. pleuropneumoniae [25]) mixed with 100,300 , or $900 \mathrm{ng}$ of competing DNA (H. influenzae KW20, A. pleuropneumoniae HS143, B. subtilis or $H$. parasuis (strain Nagasaki) DNA). DNaseI was then added at $1.0 \mu \mathrm{g} / \mathrm{ml}$ for a further five minutes and cells were then diluted and plated on supplemented BHI plates containing $2.5 \mu \mathrm{g}$.ml novobiocin (H. influenzae) or $25 \mu \mathrm{g} / \mathrm{ml} \mathrm{kan-}$ amycin (A. pleuropneumoniae). Data were plotted using the double-reciprocal method of Sisco and Smith [56].

\section{Authors' contributions}

RJR, WAF, JB and JHEN were involved in the conception and design of the study. RJR carried out the DNA-uptake and competition experiments, drafted the manuscript and produced the figures. WAF carried out most of the bioinformatics analyses. JB analyzed the $\mathrm{H}$. parasuis sequences. ADSC identified and analysed the competence genes in each species. JHEN participated in the bioinformatics analyses. All authors helped interpret the results and write the manuscript, and all read and approved the final manuscript.

\section{Acknowledgements}

Funding for this work has been provided to J.H.E.N. and W.A.F. through the National Research Council of Canada Genomics and Health Initiative, to R.J.R. through the Canadian Institutes for Health Research and National Institutes of Health Grant ROI GM607I5, and to JSK/JB from NRCBC joint S\&T. We thank Patrick Keeling for advice on phylogenetic methods, Sarah Highlander for alerting us to the USS-like sequences in $M$. haemolytica, Jan Maclnnis for $H$. parasuis DNA, and Rob Beiko and Barry Hall for helpful discussions. Assembly and preliminary annotation of the $A$. pleuropneumoniae L20 genomic sequence was done by Simon Foote. The $H$. somnus genome sequence data were produced by the US Department of Energy Joint Genome Institute [45]. Preliminary M. haemolytica sequence data was obtained from the Baylor College of Medicine Human Genome Sequencing Center website [46], supported by USDA/NRICGP grant 00-35204-9229 to Sarah Highlander and George Weinstock at the BCM-HGSC. We thank the Actinobacillus Genome Sequencing Project, and Bruce A. Roe, Fares Z. Najar, Allison Gillaspy, Sandra Clifton, Tom Ducey, Lisa Lewis and D.W. Dyer for the A. actinomycetemcomitans sequence data; this project is supported by a USPHS/NIH grant from the National Institute of Dental Research.

\section{References}

I. Chen I, Dubnau D: DNA uptake during bacterial transformation. Nat Rev Microbiol 2004, 2(3):24I-249.

2. Solomon JM, Grossman AD: Who's competent and when: regulation of natural genetic competence in bacteria. Trends Genet 1996, I2(4): I50-155.

3. Sikorski J, Teschner N, Wackernagel W: Highly different levels of natural transformation are associated with genomic subgroups within a local population of Pseudomonas stutzeri from soil. Appl Environ Microbiol 2002, 68(2):865-873.

4. Gromkova RC, Mottalini TC, Dove MG: Genetic transformation in Haemophilus parainfluenzae clinical isolates. Curr Microbiol 1998, 37(2): 123-126.

5. Sexton JA, Vogel JP: Regulation of hypercompetence in Legionella pneumophila. J Bacteriol 2004, I 86( I 2):38| 4-3825.

6. Fujise O, Lakio L, Wang Y, Asikainen S, Chen C: Clonal distribution of natural competence in Actinobacillus actinomycetemcomitans. Oral Microbiol Immunol 2004, 19(5):340-342.

7. Smith HO, Gwinn ML, Salzberg SL: DNA uptake signal sequences in naturally transformable bacteria. Res Microbiol 1999, 150(910):603-616.

8. Davidsen T, Rodland EA, Lagesen K, Seeberg E, Rognes T, Tonjum T: Biased distribution of DNA uptake sequences towards genome maintenance genes. Nucleic Acids Res 2004, 32(3): 1050-1058.

9. Smith HO, Tomb JF, Dougherty BA, Fleischmann RD, Venter JC: Frequency and distribution of DNA uptake signal sequences in the Haemophilus influenzae Rd genome. Science 1995, 269(5223):538-540.

10. Elkins C, Thomas CE, Seifert HS, Sparling PF: Species-specific uptake of DNA by gonococci is mediated by a 10 -base-pair sequence. J Bacteriol | 99|, I 73( I 2):39| |-39|3.

II. Karlin S, Mrazek J, Campbell AM: Frequent oligonucleotides and peptides of the Haemophilus influenzae genome. Nucleic Acids Res 1996, 24(2I):4263-4272.

12. Wang Y, Goodman SD, Redfield RJ, Chen C: Natural transformation and DNA uptake signal sequences in Actinobacillus actinomycetemcomitans. J Bacteriol 2002, 184(I3):3442-3449.

13. Bakkali M, Chen TY, Lee HC, Redfield RJ: Evolutionary stability of DNA uptake signal sequences in the Pasteurellaceae. Proc Natl Acad Sci U S A 2004, I 0 I( I3):45 I 3-45 I 8.

14. Barouki R, Smith HO: Initial steps in Haemophilus influenzae transformation. Donor DNA binding in the com 10 mutant. J Biol Chem 1986, 26 I(19):8617-8623. 
15. Danner DB, Deich RA, Sisco KL, Smith HO: An eleven-base-pair sequence determines the specificity of DNA uptake in Haemophilus transformation. Gene 1980, I I(3-4):3 I I-3 I8.

16. Christensen H, Foster G, Christensen JP, Pennycott T, Olsen JE, Bisgaard M: Phylogenetic analysis by I6S rDNA gene sequence comparison of avian taxa of Bisgaard and characterization and description of two new taxa of Pasteurellaceae. J Appl Microbiol 2003, 95(2):354-363.

17. Christensen H, Kuhnert $\mathrm{P}$, Olsen JE, Bisgaard M: Comparative phylogenies of the housekeeping genes atpD, infB and rpoB and the I6S rRNA gene within the Pasteurellaceae. Int J Syst Evol Microbiol 2004, 54(Pt 5): I60I-1609.

18. Dewhirst FE, Paster BJ, Olsen I, Fraser GJ: Phylogeny of 54 representative strains of species in the family Pasteurellaceae as determined by comparison of I 6S rRNA sequences. J Bacteriol 1992, I 74(6):2002-2013.

19. Kilian M: Taxonomic Study of Genus Hemophilus, with Proposal of a New Species. Journal of General Microbiology 1976 93(MAR):9-62.

20. Norskov-Lauritsen N, Bruun B, Kilian M: Multilocus sequence phylogenetic study of the genus Haemophilus with description of Haemophilus pittmaniae sp. nov. Int J Syst Evol Microbiol 2005, 55(Pt I):449-456.

21. Lee PC, Lee SY, Hong SH, Chang HN: Isolation and characterization of a new succinic acid-producing bacterium, Mannheimia succiniciproducens MBEL55E, from bovine rumen. Appl Microbiol Biotechnol 2002, 58(5):663-8.

22. Hong SH, Kim JS, Lee SY, In YH, Choi SS, Rih JK, Kim CH, Jeong H, Hur CG, Kim J]: The genome sequence of the capnophilic rumen bacterium Mannheimia succiniciproducens. Nat Biotechnol 2004, 22(I 0): | 275-I28I.

23. Hong SH, Kim JS, Lee SY, In YH, Choi SS, Rih JK, Kim CH, Jeong $\mathrm{H}$ Hur CG, Kim J]: Corrigendum: The genome sequence of the capnophilic rumen bacterium Mannheimia succiniciproducens. Nat Biotechnol 2004, 22( I 2): I 589.

24. Alexander $\mathrm{H}$, Leidy $\mathrm{G}$ : Determination of inherited traits of $\mathbf{H}$ influenzae by desoxyribonucleic acid fractions isolated from type-specific cells. J Exp Med I95I, 93:345-359.

25. Bosse JT, Nash JH, Kroll JS, Langford PR: Harnessing natural transformation in Actinobacillus pleuropneumoniae: a simple method for allelic replacements. FEMS Microbiol Lett 2004, 233(2):277-28।.

26. Bigas A, Garrido ME, Rozas AM, Badiola I, Barbe J, Llagostera M: Development of a genetic manipulation system for Haemophilus parasuis. Vet Microbiol 2005, I 05(3-4):223-228.

27. Nickel L, Goodgal SH: Effect of Interspecific Transformation on Linkage Relationships of Markers in Haemophilus influenzae and Haemophilus parainfluenzae. I Bacteriol 1964, 88: $1538-1544$

28. VanWagoner TM, Whitby PW, Morton DJ, Seale TW, Stull TL: Characterization of three new competence-regulated operons in Haemophilus influenzae. J Bacteriol 2004, I 86( I 9):6409-642 I.

29. Redfield RJ, Cameron ADS, Qian Q, Hinds J, Ali TR, Kroll JS, Langford PR: A novel CRP-dependent regulon controls expression of competence genes in Haemophilus influenzae. J Mol Biol 2005 347(4):735-747.

30. May BJ, Zhang Q, Li LL, Paustian ML, Whittam TS, Kapur V: Complete genomic sequence of Pasteurella multocida, Pm70. Proc Natl Acad Sci U S A 200I, 98(6):3460-3465.

31. Ochman $\mathrm{H}$ : Neutral mutations and neutral substitutions in bacterial genomes. Mol Biol Evol 2003, 20(I 2):209|-2096.

32. Lethem MI, James SL, Marriott C, Burke JF: The origin of DNA associated with mucus glycoproteins in cystic fibrosis sputum. Eur Respir J 1990, 3(I): 19-23.

33. Crooks GE, Hon G, Chandonia JM, Brenner SE: WebLogo: a sequence logo generator. Genome Res 2004, I 4(6): I I 88- I I 90.

34. Melnikow E, Dornan S, Sargent C, Duszenko M, Evans G, Gunkel N, Selzer PM, Ullrich HJ: Microarray analysis of Haemophilus parasuis gene expression under in vitro growth conditions mimicking the in vivo environment. Vet Microbiol 2005, I I 0(3 4):255-263.

35. White DC, Leidy G, Jamieson JD, Shope RE: Porcine Contagious Pleuropneumonia. 3. Interrelationship Of Hemophilus pleuropneumoniae To Other Species Of Hemophilus: Nutritional, Metabolic, Transformation, And Electron Microscopy Studies. J Exp Med 1964, 120:1-12.
36. Scocca J], Poland RL, Zoon KC: Specificity in deoxyribonucleic acid uptake by transformable Haemophilus influenzae. J Bacteriol 1974, I I 8(2):369-373.

37. Albritton WL, Setlow JK, Thomas ML, Sottnek FO: Relatedness within the family Pasteurellaceae as determined by genetic transformation. Int J Syst Bact 1986, 36(I): I03-106.

38. Kahn ME, Barany F, Smith HO: Transformasomes: specialized membranous structures that protect DNA during Haemophilus transformation. Proc Natl Acad Sci U S A 1983, 80(22):6927-6931.

39. Setlow JK, Boling ME, Allison DP, Beattie KL: Relationship between prophage induction and transformation in Haemophilus influenzae. Journal of Bacteriology 1973, I I 5(I): I53-I6I.

40. Tanaka MM, Bergstrom CT, Levin BR: The evolution of mutator genes in bacterial populations: the roles of environmenta change and timing. Genetics 2003, I 64(3):843-854

41. Harrison A, Dyer DW, Gillaspy A, Ray WC, Mungur R, Carson MB, Zhong H, Gipson J, Gipson M, Johnson LS, Lewis L, Bakaletz LO, Munson RSJ: Genomic sequence of an otitis media isolate of nontypeable Haemophilus influenzae: comparative study with H. influenzae serotype d, strain KW20. J Bacteriol 2005 I 87( I3):4627-4636.

42. Fleischmann RD, Adams MD, White O, Clayton RA, Kirkness EF, Kerlavage AR, Bult CJ, Tomb JF, Dougherty BA, Merrick JM, et al.: Whole-genome random sequencing and assembly of Haemophilus influenzae Rd. Science 1995, 269(5223):496-5I 2.

43. National Center for Biotechnology Information [http:// www.ncbi.nlm.nih.gov]

44. Actinobacillus actinomycetemcomitans Strain HKI65I Genome Sequencing [http://www.genome.ou.edu/act.html]

45. DOE Joint Genome Institute [http://www.jgi.doe.gov]

46. Baylor College of Medicine Human Genome Sequencing Center [http://www.hgsc.bcm.tmc.edu]

47. Gioia J, Qin X, Jiang H, Clinkenbeard K, Lo R, Liu Y, Fox GE Yerrapragada S, McLeod MP, McNeill TZ, Hemphill L, Sodergren E, Wang Q, Muzny DM, Homsi FJ, Weinstock GM, Highlander SK: The Genome Sequence of Mannheimia haemolytica AI: Insights into Virulence, Natural Competence, and Pasteurellaceae Phylogeny. J Bacteriol 2006, I 88(20):7257-7266.

48. Glimmer Microbial Gene Finding System [http:// cbcb.umd.edu/software/glimmer]

49. Altschul SF, Gish W, Miller W, Myers EW, Lipman DJ: Basic loca alignment search tool. J Mol Biol I990, 2 I 5(3):403-4I0.

50. Thompson JD, Higgins DG, Gibson TJ: CLUSTAL W: improving the sensitivity of progressive multiple sequence alignment through sequence weighting, position-specific gap penalties and weight matrix choice. Nucleic Acids Res 1994 22(22):4673-4680.

51. Felsenstein J: PHYLIP - Phylogeny inference package (version 3.2). Cladistics 1989, 5:164-166

52. NRC Institute for Biological Sciences: Genomics and Proteomics [http://ibs-isb.nrc-cnrc.gc.ca/glycobiology/ group software e.html]

53. WebLogo [http://weblogo.berkeley.edu

54. Poje G, Redfield RJ: Transformation of Haemophilus influenzae. In Haemophilus influenzae Protocols Edited by: Herbert MA, Hood DW, Moxon ER. Totowa NJ , Humana; 2003:57-70.

55. Poje G: DNA uptake specificity of Haemophilus influenzae. In Zoology Vancouver , University of British Columbia; 2000.

56. Sisco KL, Smith HO: Sequence-specific DNA uptake in Haemophilus transformation. Proc Natl Acad Sci U S A 1979 76(2):972-976.

57. Lerat E, Daubin V, Moran NA: From gene trees to organismal phylogeny in prokaryotes: the case of the gamma-Proteobacteria. PLoS Biol 2003, I(I):EI9. 